\title{
Magnesium as a biomaterial and its biological interactions
}

\author{
Evertz F. ${ }^{1}$, Hauser H. ${ }^{2}$, Müller P.P. ${ }^{2}$, Kietzmann M. ${ }^{3}$, Maier H.J. ${ }^{4}$, Glasmacher B. ${ }^{1}$ \\ ${ }^{1}$ Institute for Multiphase Processes, Leibniz Universität Hannover, Germany \\ ${ }^{2}$ Helmholz Centre for Infection Research, Braunschweig, Germany \\ ${ }^{3}$ University of Veterinary Medicine, Hannover, Germany \\ ${ }^{4}$ Institute of Material Science, Leibniz Universität Hannover, Germany
}

evertz@imp.uni-hannover.de

\begin{abstract}
To investigate different magnesium alloys as a biodegradable material for implants it is necessary to understand the degradation process in the biological environment. The following study shows interactions of pure magnesium with various physical and chemical environmental parameters according to the parameters within the application area.
\end{abstract}

Keywords: Mg degradation, implant, biomaterial, in vitro

\section{Introduction}

Magnesium is studied as bio corroding implant material. The in-vitro degradation shows different kinetics compared to the degradation in vivo. Daters published in literature are not comparable because of different degradation systems and measuring parameters. The aim of this study is to describe the interactions and the behaviour of pure magnesium in different fluid systems and under different physical conditions. Thus we would like to be able to predict the in vivo corrosion with in vitro data.

\section{Methods}

Literature data show different model fluids to simulate the in vivo degradation system in vitro. As a result, the degradation kinetics cannot be compared because of different components in the fluids that have an impact on the corrosion process. Fig 1 shows the model fluids described in literature used for in vitro degradation studies.



Figure 1: Model fluids and their use in in vitro studies.

For our studies we chose the mostly used model fluids to test their influence on the Mg degradation. In addition to that, we used porcine serum to be able to compare the results with a biological fluid. Table 1 shows the ionic compositions of the different fluids. The first degradation studies were done under static conditions and executed as a standard immersion test. Therefore the samples were immersed in $10 \mathrm{ml}$ of each model fluid for up to 18 days. The fluid was exchanged every 24 hours and analysed by measuring the $\mathrm{pH}$ value and the Mg ion concentration in the fluid. Pure $\mathrm{Mg}$ - all samples used in this study origin from one batch in order to minimize the influence of different grain boundaries or possible contaminations on the degradation. Table 2 shows an ICP OES measurement; the data illustrate the purity of the material we used. The sample geometry chosen is a cylinder with a thickness of $2 \mathrm{~mm}$ and a diameter of $5 \mathrm{~mm}$. The study is performed at $37^{\circ} \mathrm{C}$, group A with and group B without $\mathrm{CO}_{2}$.

Table 1: Ionic composition of the model fluids

\begin{tabular}{l|lllll} 
[mMol/l] & serum & NaCl & c-SBF & r-SBF & m-SBF \\
\hline Na & 142 & 153 & 142 & 142 & 142 \\
K & 5 & & 5 & 5 & 5 \\
Mg & 1,5 & & 1,5 & 1,5 & 1,5 \\
Ca & 2,5 & & 2,5 & 2,5 & 2,5 \\
Cl & 103 & 153 & 147,8 & 103 & 103 \\
HCO3 & 27 & & 4,2 & 27 & 10 \\
HPO4 & 1 & & 1 & 1 & 1 \\
SO4 & 0,5 & & 0,5 & 0,5 & 0,5
\end{tabular}

A new dynamic in vitro test device as shown in figure 2 has been designed to investigate the influence of physical flow conditions on the degradation process. This system is able to mimic different flow characteristic such as pulsatile flow with different flow rates.

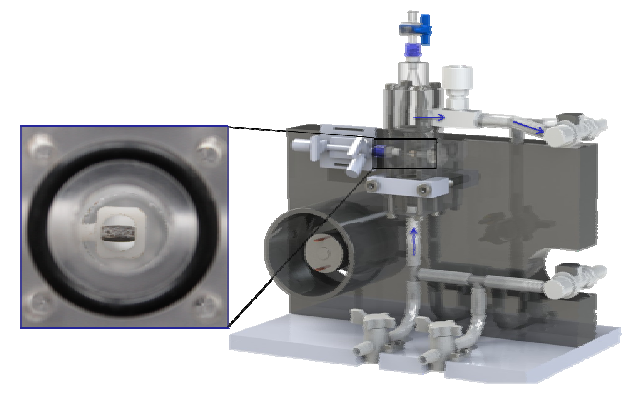

Figure 2: Dynamic in vitro degradation device.

Table 2: ICP-OES contaminations of the Mg samples

\begin{tabular}{l|l} 
element & amount \\
\hline $\mathbf{F e}$ & $0,7[\mu \mathrm{g} / \mathrm{l}]$ \\
$\mathbf{N i}$ & $-[\mathrm{mg} / \mathrm{l}]$ \\
$\mathbf{C u}$ & $-[\mathrm{mg} / \mathrm{l}]$ \\
$\mathbf{M n}$ & $0,012[\mathrm{mg} / \mathrm{l}]$
\end{tabular}

Within the second study, the Mg samples were located in flow direction in the middle of the test chamber. Thus, the model fluid flow was directed against the thin site of the sample (Figure 2). The samples were exposed to saline at flow 
velocities of $1,3 \mathrm{~mm} / \mathrm{s}, 3,3 \mathrm{~mm} / \mathrm{s}$ and $6,6 \mathrm{~mm} / \mathrm{s}$ respectively. Weight loss and concentration of $\mathrm{Mg}^{2+}$ in the solution were measured to characterise the rate of degradation.

\section{Results}

The degradation rate depends directly on the composition of the model fluid which is used in the degradation test. Figure 2 shows the results of the degradation process in four different fluids. The data illustrate that only by changing the fluid, the rate of degradation changes.

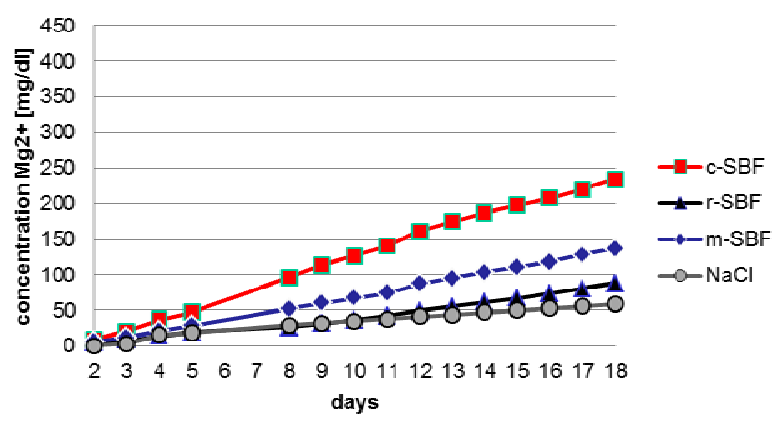

Figure 3: Degradation in different fluids without CO2 (n=3)

In the case of c- and r SBF; these solutions only differ in the amount of chloride and $\mathrm{HCO}_{3}{ }^{-}$, the degradation of cSBF is three times faster than that of r-SBF. The low rate of degradation in $\mathrm{NaCl}$ solution is due to $\mathrm{pH}$ change. The same test is performed with $\mathrm{CO} 2$ control to stabilize the buffer stability and thus keep the $\mathrm{pH}$ value constant. In this study we also investigated porcine blood serum as a model fluid. Figure 4 shows that the degradation rate increases only by gassing the fluid with $\mathrm{CO}_{2}$. This results in a stable $\mathrm{pH}$ value over the test period.

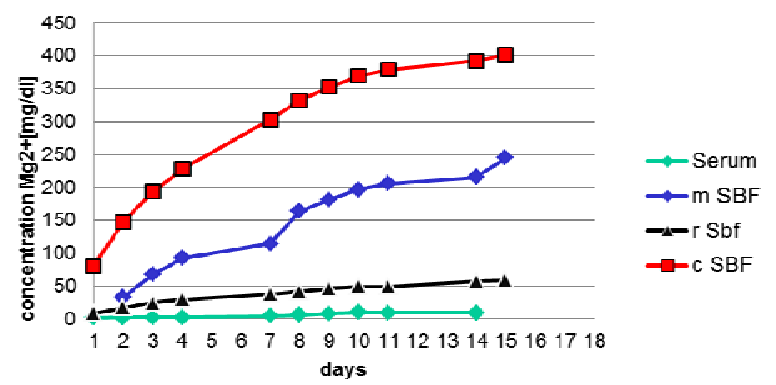

Figure 4: Degradation in different fluids with CO2 (n=3).

Porcine serum lead to the lowest degradation rate within our study. With respect to the ion composition of r-SBF there is no difference between these two solutions. The influencing factor of this solution seems to be the amount of proteins. Figure 4 exemplarily depicts an EDX spectrum of a magnesium sample in serum. The spectrum of this serum immersed $\mathrm{Mg}$ in comparison to r-SBF Mg shows a high amount of carbon on the sample surface. This C-signal is an indication of a protein layer formation on the sample surface which has a passivation effect on the Mg. The EDX spectrum of r-SBF Mg also reveals a small C-peak, but is related to the sample fabrication process. The dynamic corrosion study shows an increased corrosion by increasing the test fluid velocity (Figure 6). Pitting corrosion is found in the area with a direct exposure to the fluid flow as depicted in figure 7.

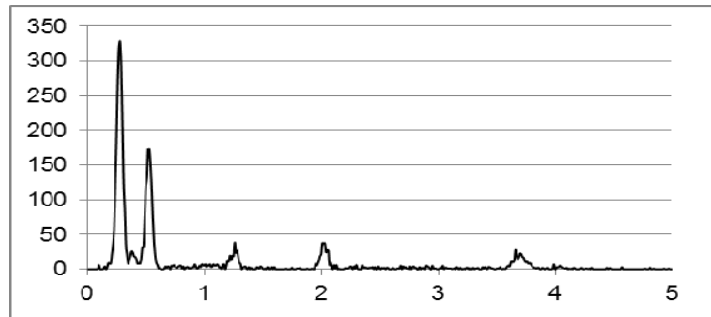

Figure 5: Degradation in different model fluids without CO2

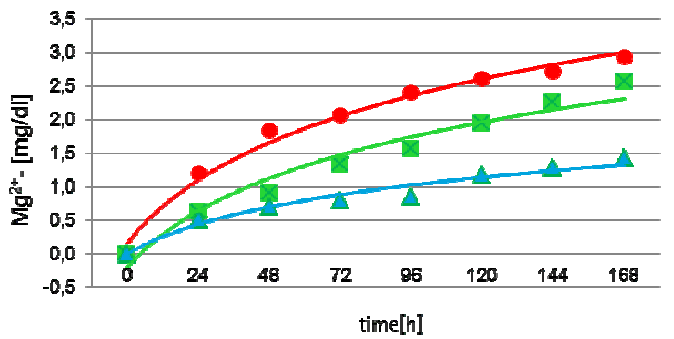

Figure 6: Dynamic degradation of $\mathrm{Mg}$ samples in saline solution $(n=5)$
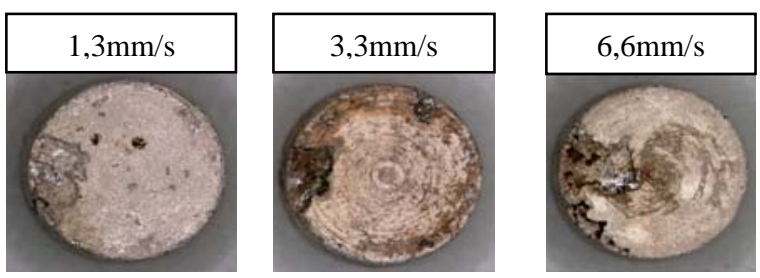

Figure 5: Light microscopic pictures of magnesium samples studied with different fluid flow velocities.

\section{Discussion}

The study shows the importance of knowing the influence of the chosen model fluid on the degradation process. To be able to compare experimental data, we need to know the exact composition of the degradation fluids used in different studies. Furthermore, the data shown in this study demonstrate the possibility to design in vitro studies that resemble the in vivo situation by choosing the appropriate degradation fluid and physical parameters. Dynamic flow models seem to be superior to just static incubation tests. The degradation of magnesium in protein containing solutions revealed a slower and decreased degradation process. The primary reason for that seems to be the formation of a protein layer on the surface of the sample. In further studies the mechanism of the degradation protection by the protein layer will be investigated; one aspect will be to find out the kind of protein with highest impact on the protection.

\section{Acknowledgement}

The authors would like to thank the German Research Foundation (DFG) for the financial support of this study within SFB 599 "Sustainable bioresorbable and permanent implants of metallic and ceramic materials”. 
